GSA Data Repository 2016023

\title{
An apatite for progress: Inclusions in zircon and titanite
}

\section{constrain petrogenesis and provenance}

\author{
Emilie Bruand, Craig Storey, and Mike Fowler
}

\section{A- Sample locations}

\begin{tabular}{lll} 
& Rock Type & Grid Reference \\
\hline Strontian & & \\
\hline SR1 & Granodiorite & NM795611 \\
SR3 & Granodiorite & NM779607 \\
SR4 & Granodiorite & NM835655 \\
\hline Rogart & & NC671046 \\
\hline RHG-1 & Granite & NC709029 \\
R2 & Tonalite & NC741065
\end{tabular}

The grid referencing system used is the Ordnance Survey National Grid reference system using OSGB 36 datum

\section{B- Analytical Techniques}

The samples were crushed (jaw-crusher, ball mill or Selfrag $\left.{ }^{\mathrm{TM}}\right)$, sieved $(<355 \mu \mathrm{m}$, 355-500 $\mu \mathrm{m}$ and 500-1000 $\mu \mathrm{m}$ fractions) and passed over a Wilfley table. A diamagnetic separator was then used to obtain fractions of different heavy minerals based on their diamagnetic properties. Titanite, apatite and zircon were handpicked, mounted in epoxy resin discs and polished for in-situ chemical analysis. Titanite and apatite have also been analysed within thick sections (c.150 $\mu \mathrm{m})$. Back-scattered electron (BSE) images of titanites were taken with a scanning electron microscope (SEM) JEOL JSM-6100 at the University of Portsmouth 
(accelerating voltage $=20 \mathrm{kV}$ ). Cathodoluminescence $(\mathrm{CL})$ images of apatites and zircons were taken with a KeDev Centaurus cathodoluminescence detector housed within a JEOL 6060LV SEM also at the University of Portsmouth (accelerating voltage $=15 \mathrm{kV}$ ).

\section{Electron probe microanalysis (EPMA)}

A Cameca SX-100 microprobe at Bristol University was used for determination of major elements in titanite and zircon using TAP, LPET, PET and LLIF crystals. PC0, TAP, LPET and LLIF crystals were used for apatite. An electron beam of $1 \mu \mathrm{m}$ was used for titanite and $10 \mu \mathrm{m}$ for apatite, both with $20 \mathrm{kV}$ accelerating voltage and, 40nA and $10 \mathrm{nA}$ beam currents respectively. An electron beam of $5 \mu \mathrm{m}$ was used for zircon with an accelerating voltage of $17 \mathrm{kV}$ and a beam current of $100 \mathrm{nA}$. Several trace elements in these minerals were also analysed for comparison with laser ablation ICP-MS (LA-ICPMS) data. The Durango apatite standard (Marks et al., 2012) and the 91500 zircon standard (Wiedenbeck et al., 2004) were analysed during sessions to monitor data quality.

\section{Ion microprobe}

Apatite inclusions were analysed by ion microprobe (Cameca ims 4f) at the NERC Ion Microprobe Facility in Edinburgh using a 15kV Accelerating voltage and 1nA to 2 nA primary beam corresponding to an approximately 15 microns spot size. Positive secondary ions of $120 \mathrm{~V}$ energy were measured with a $40 \mathrm{~V}$ window to suppress molecular ions. Rare Earth Elements (REE), V, Mn, Th and U were based on NIST 610 (Pearce et al., 1997). F and Cl were based on a dataset of apatite standards (Steve Bergman personal communication) and Durango apatite using EPMA data. Reference Durango apatite (Marks et al., 2012) was used as a secondary standard. $\mathrm{CaO}$ determined by EPMA on apatite matrix was used as the internal standard.Variation of $\mathrm{CaO}$ content in apatite from the matrix are within the error of the $4 \mathrm{f}$ 
analysis. Three corrections were made (i) for light REE oxides on the heavy REE using $\mathrm{CeO} / \mathrm{Ce}$ ratios and tables of REEO/REE previously established for apatites, (ii) correction for CeF on $\mathrm{Tb}$ were based on $\mathrm{CaF} / \mathrm{Ca}$, (iii) correction for ion yield for REE was based on the ion yield vs Ca content difference between NIST 610 value and Durango apatite.

Uncertainty based on the detection limit of $\mathrm{Yb}$ is high and can be seen by highly scattered $\mathrm{Yb}$ concentrations at low beam current. Therefore $\mathrm{Yb}$ has been removed from the presented analysis. Details of Durango analyses and their comparison with literature data can be found in the Table DR1. Accuracy and precision on the ion microprobe data have been assessed by repeated measurements of our Durango standard (Table DR1).

\section{Laser ablation-inductively coupled plasma mass spectrometry (LA-ICPMS)}

Trace element contents of titanite, matrix apatite and zircon were analysed by LA-ICPMS at the University of Portsmouth using an Agilent 7500cs (quadrupole) ICPMS and a New-Wave UP213 ( $\lambda=213 \mathrm{~nm}$ ) solid state Nd:YAG laser. Each analysis consisted of ca. $30 \mathrm{~s}$ background acquisition and $60 \mathrm{~s}$ sample acquisition. The diameter of the laser beam was $30 \mu \mathrm{m}$ for titanite and apatite and either $30 \mu \mathrm{m}$ or $40 \mu \mathrm{m}$ for zircon. Each analytical run had either 9 or 16 spot analyses with at least two external standard analyses at the beginning and end of each run (either NIST 610 or NIST 612). NIST 610 standard (Pearce et al., 1997) was analysed with a laser beam diameter of $55 \mu \mathrm{m}$ before and after titanite and apatite unknown runs. In addition, Durango apatite (reference value used: Marks et al., 2012) was also analysed as a secondary standard with a laser beam diameter of $30 \mu \mathrm{m}$ at the beginning of each apatite run. Internal references used for normalisation of LA-ICPMS data were ${ }^{43} \mathrm{Ca}$ for apatite and titanite, and ${ }^{29}$ Si for zircon and were obtained by EPMA. Details of Durango and NIST 610 analyses and their comparison with literature data can be found in Bruand et al. (2014). Accuracy and precision on the LA-ICPMS data have been assessed by repeated 
measurements of our NIST 610 standard.

Bruand, E., Storey, C. and Fowler, M., 2014, Accessory mineral chemistry of High Ba-Sr granites from northern Scotland: constrains on petrogenesis and record of whole-rock signature: Journal of Petrology, DOI: 10.1093/petrology/egu037 (in press).

Marks, M.A.W., Wenzel, T., Whitehouse, M.J., Loose, M., Zack, T., Barth, M., Worgard, L., Krasz, V., Eby, G.N., Stosnach, H., and Markl, G., 2012, The volatile inventory (F, $\mathrm{Cl}, \mathrm{Br}, \mathrm{S}, \mathrm{C}$ ) of magmatic apatite: An integrated analytical approach: Chemical Geology, v. 291, p. 241-255.

Pearce, N.J.G., Perkins, W.T., Westgate, J.A., Gorton, M.P., Jackson, S.E., Neal, C.R., and Chenery, S.P., 1997, A compilation of new and published major and trace element data for NIST SRM 610 and NIST SRM 612 glass reference materials: Geostandards Newsletter-the Journal of Geostandards and Geoanalysis, v. 21, p. 115-144.

Wiedenbeck, M., Hanchar, J.M., Peck, W.H., Sylvester, P., Valley, J., Whitehouse, M., Kronz, A., Morishita, Y., Nasdala, L., Fiebig, J., Franchi, I., Girard, J.P., Greenwood, R.C., Hinton, R., Kita, N., Mason, P.R.D., Norman, M., Ogasawara, M., Piccoli, R., Rhede, D., Satoh, H., Schulz-Dobrick, B., Skar, O., Spicuzza, M.J., Terada, K., Tindle, A., Togashi, S., Vennemann, T., Xie, Q., and Zheng, Y.F., 2004, Further characterisation of the 91500 zircon crystal: Geostandards and Geoanalytical Research, v. 28, p. 9-39. 

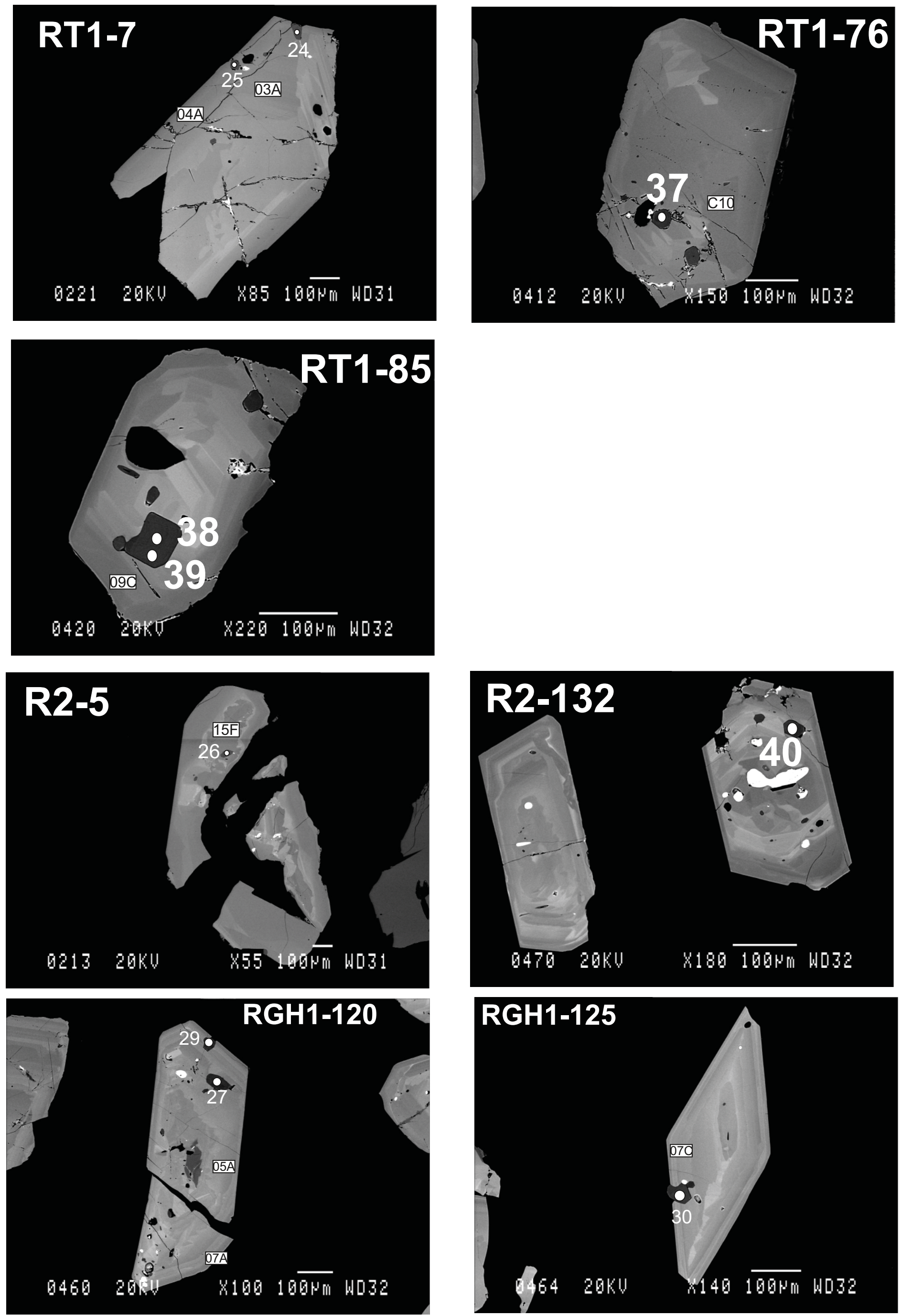

Fig. DR1-A 

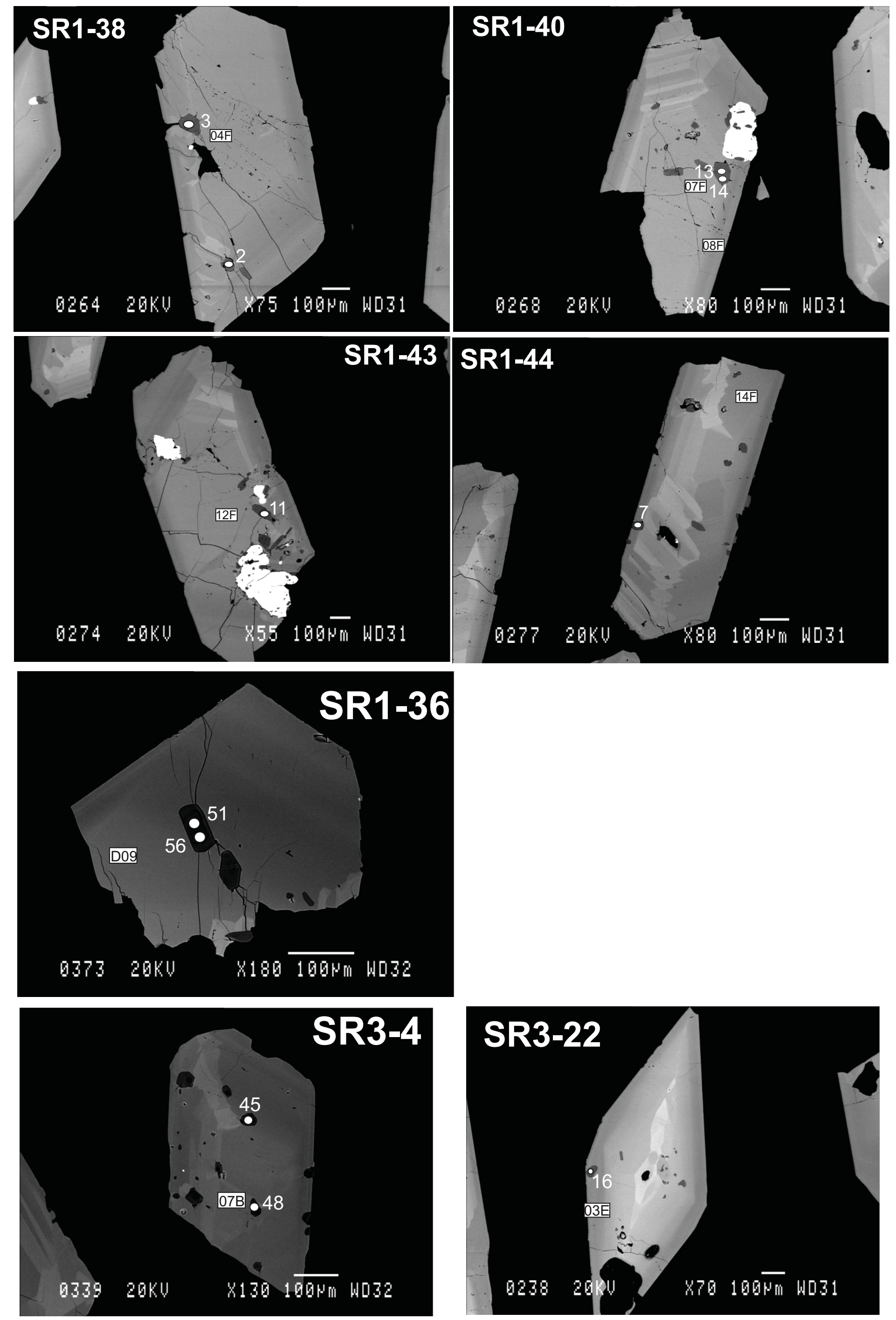

Fig. DR1-B 


\section{SR3-10}
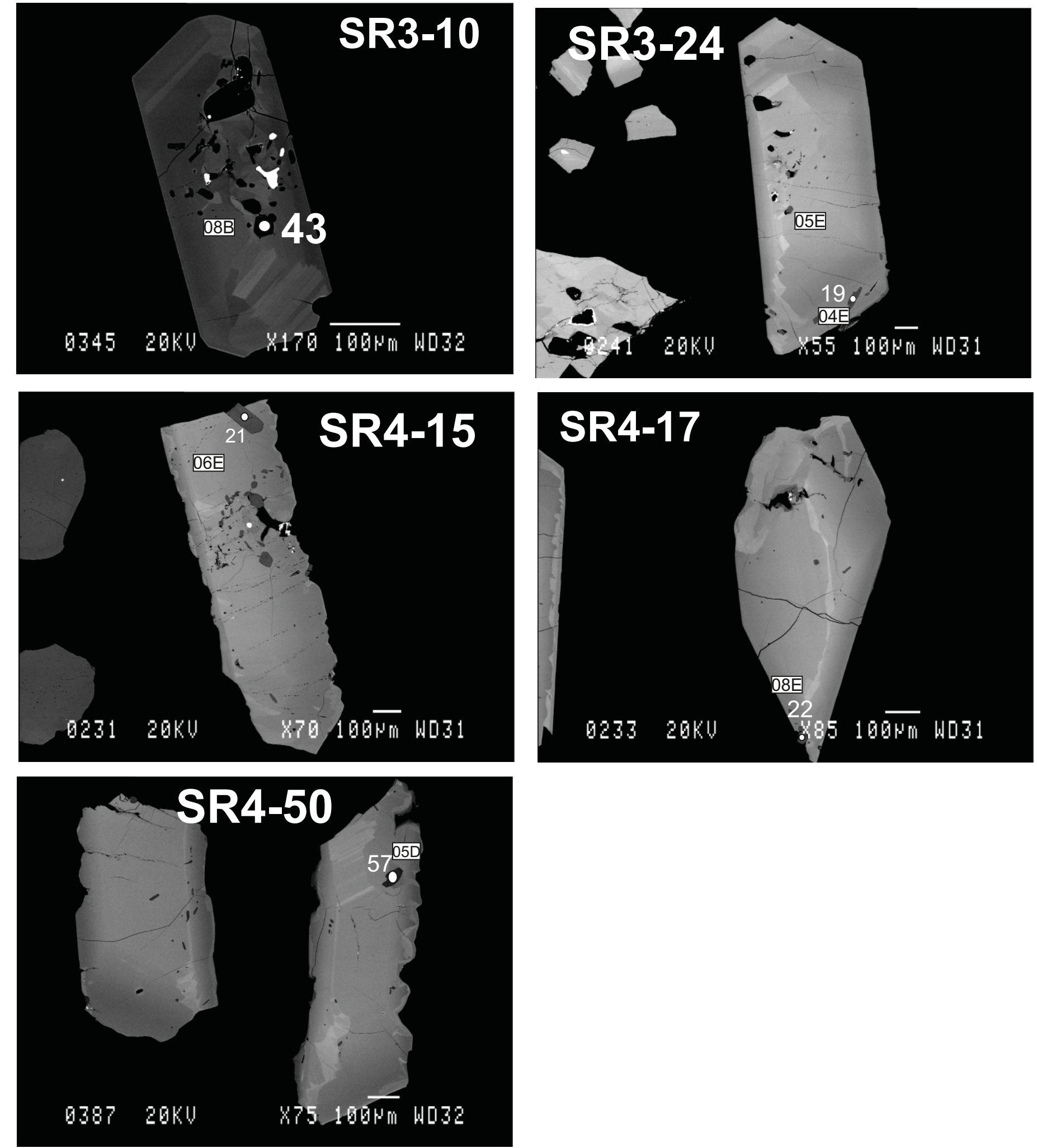

Fig. DR1-C 

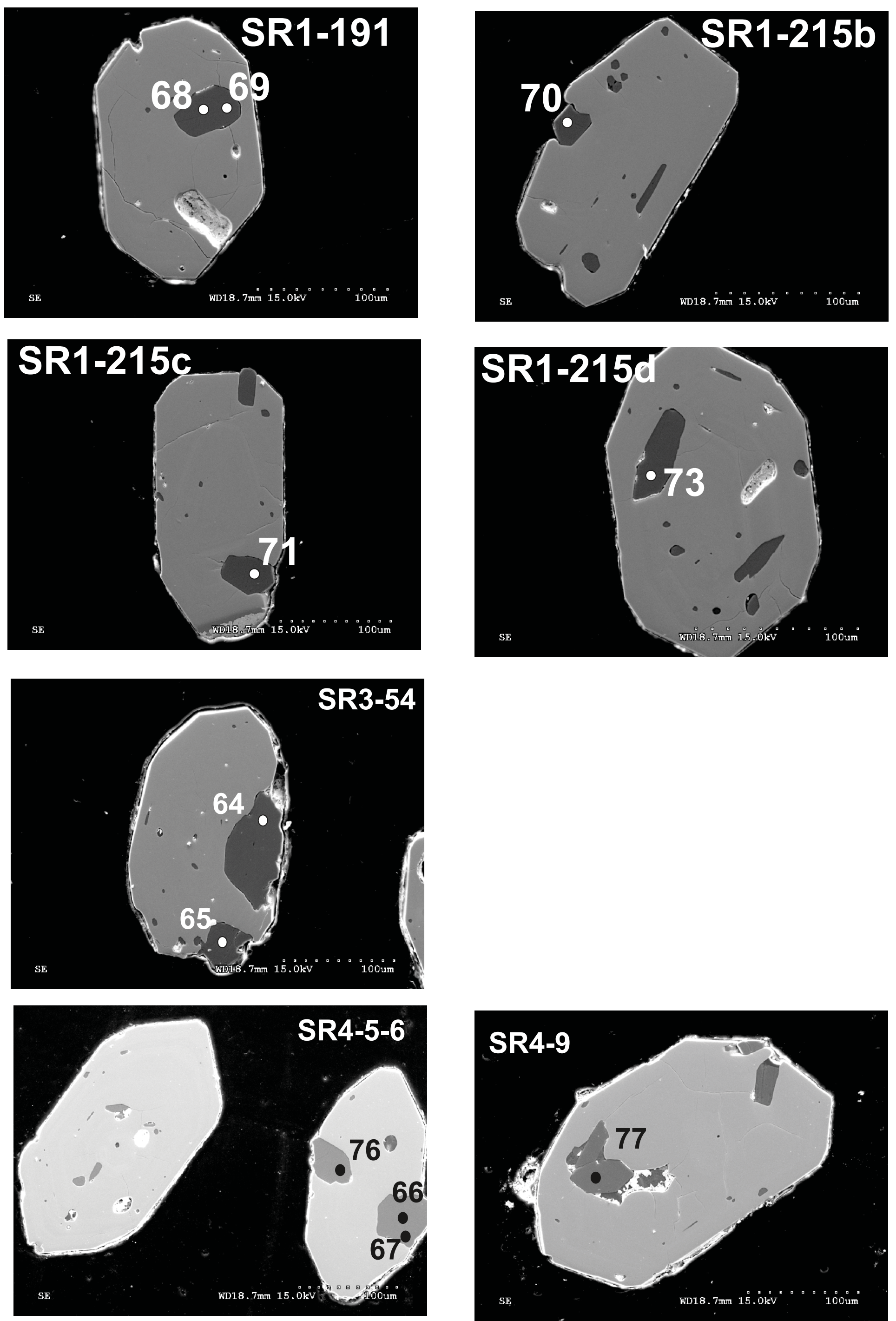

Fig. DR1-D 

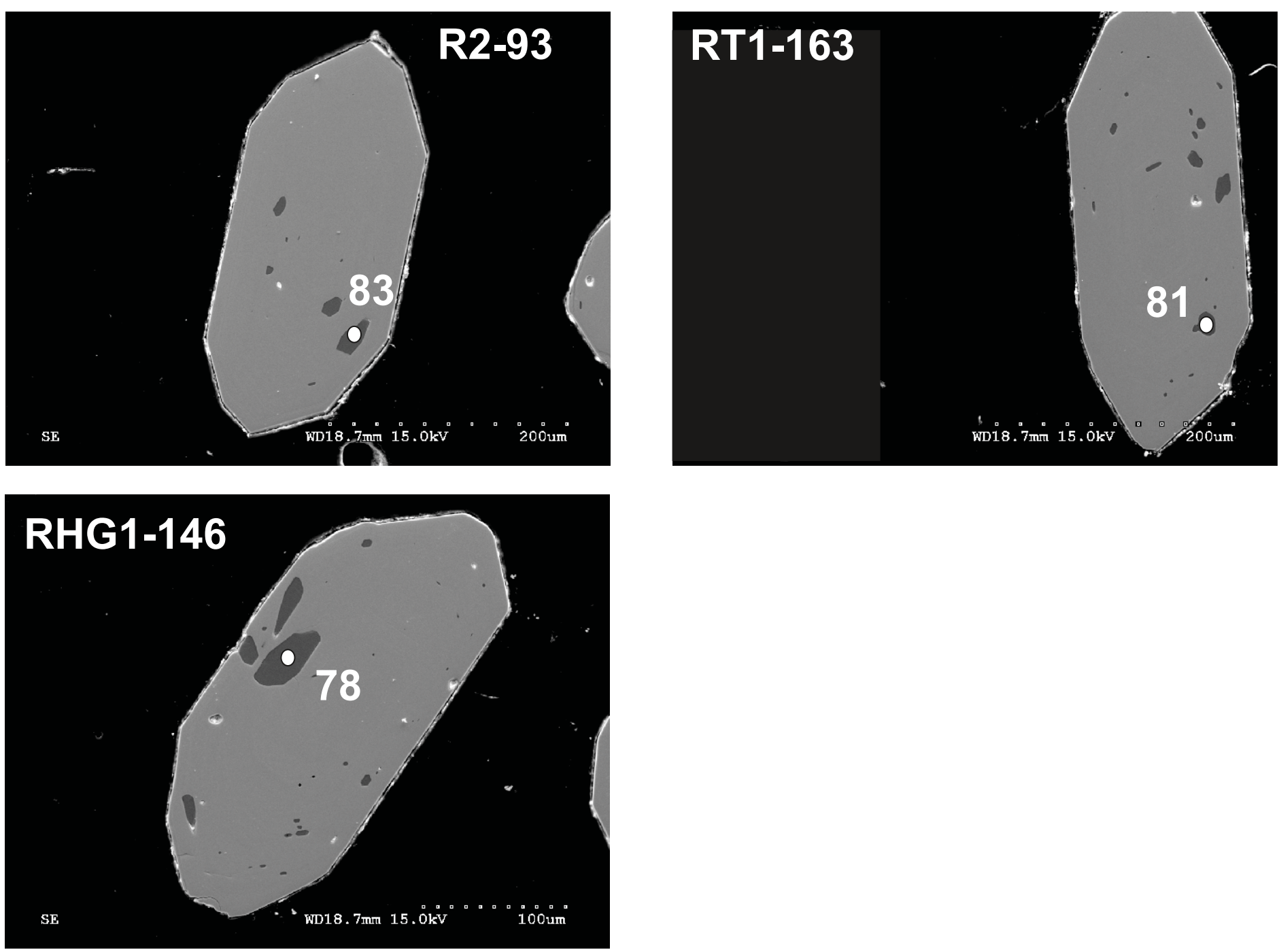

Fig. DR1-E 

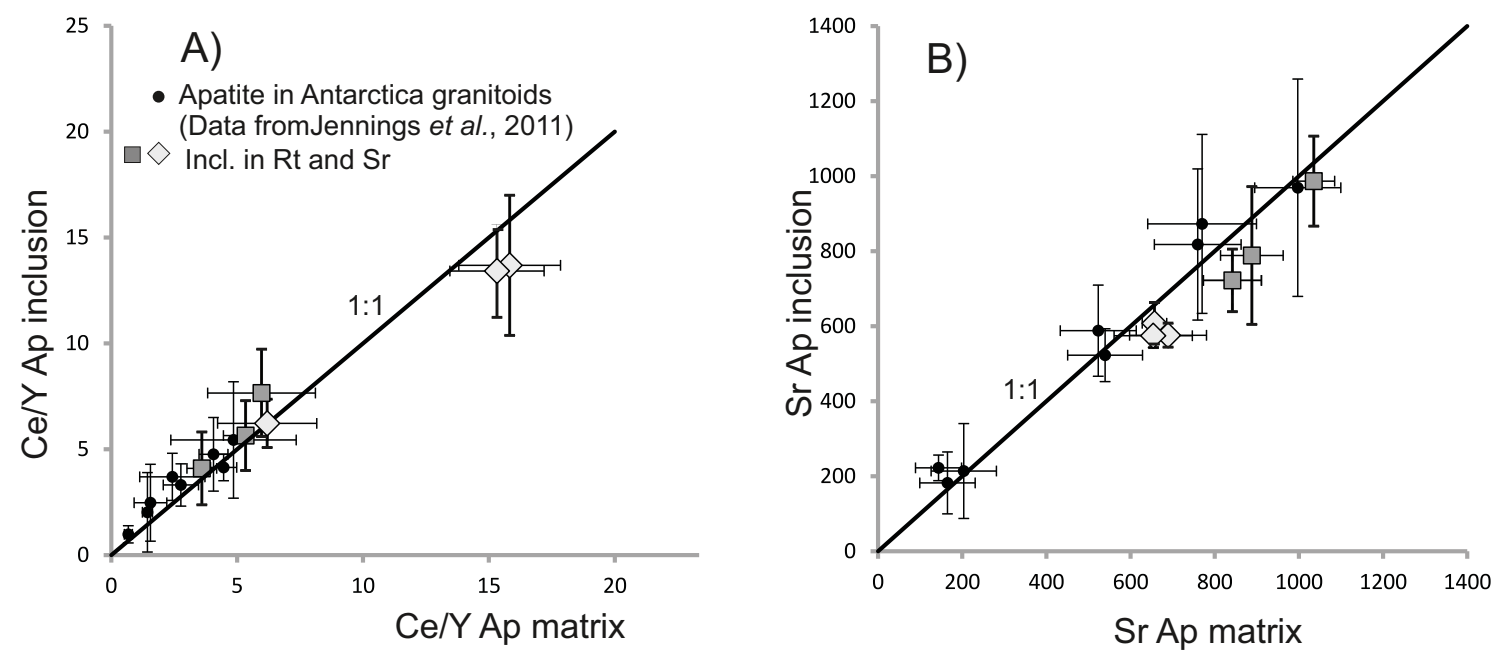

Fig. DR2 

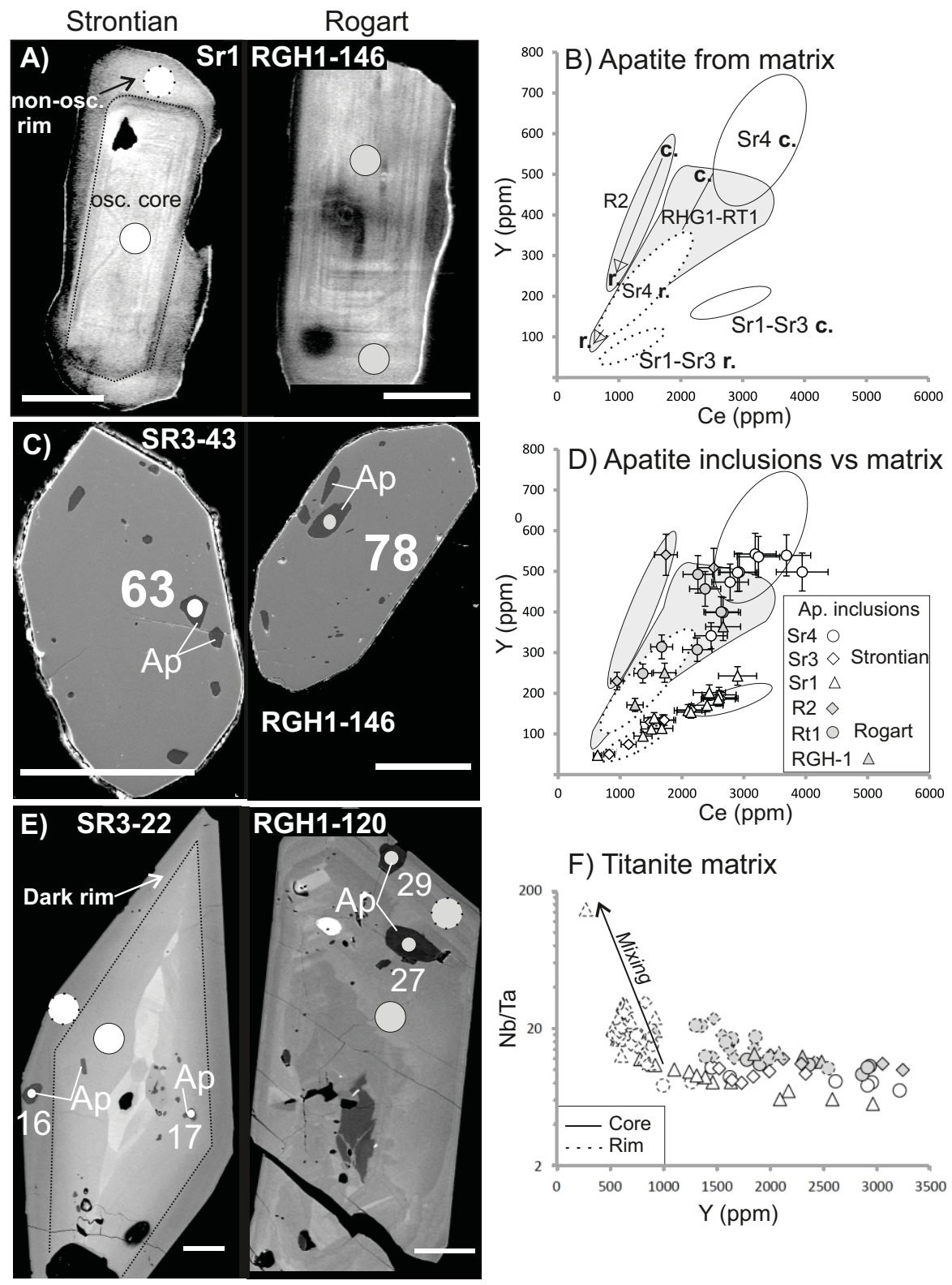

F) Titanite matrix

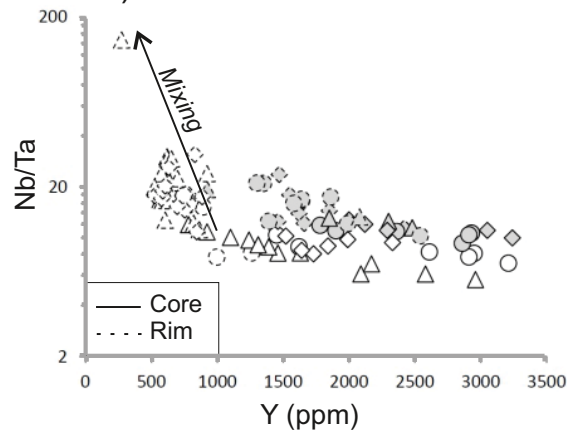

Fig. DR3 
Table DR1- 1 Analysis of apatite inclusions compositions and Durango standard using the Cameca ims 4 at Edinburgh. $R=$ apatite in rim, ft=apatite in fir-tree zoning, cr= inclusions close by cracks. F, SiO2, P2O5, $\mathrm{Cl}$ and $\mathrm{CaO}$ are in wt\%. Other elements are in ppm.

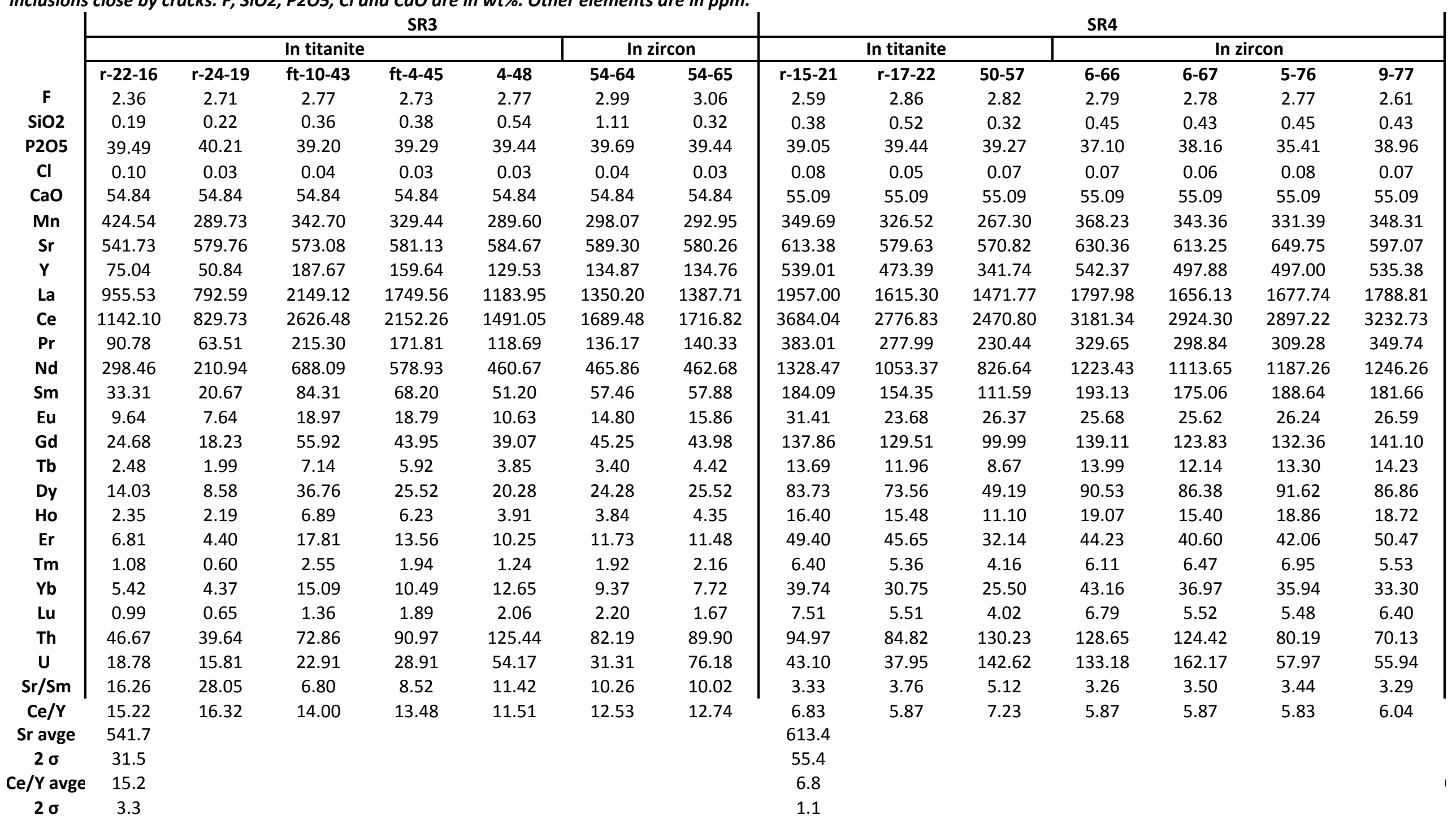




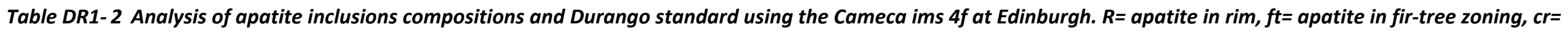
inclusions close by cracks. $\mathrm{F}, \mathrm{SiO2}, \mathrm{P2O}, \mathrm{Cl}$ and $\mathrm{CaO}$ are in wt\%. Other elements are in ppm.

\begin{tabular}{|c|c|c|c|c|c|c|c|c|c|c|c|c|c|}
\hline & \multicolumn{13}{|c|}{ SR1 } \\
\hline & \multicolumn{8}{|c|}{ In titanite } & \multicolumn{5}{|c|}{ In zircon } \\
\hline & ft-cr38-2 & cr-38-3 & $r-44-7$ & cr-43-11 & $r-40-13$ & $r-40-14$ & cr-36-51 & cr-36-56 & $191-68$ & $191-69$ & $215 b-70$ & $215 c-71$ & $215 d-73$ \\
\hline $\mathbf{F}$ & 2.71 & 2.72 & 3.04 & 2.68 & 2.87 & 2.85 & 2.72 & 2.84 & 2.97 & 2.87 & 2.55 & 2.77 & 2.82 \\
\hline $\mathrm{SiO2}$ & 0.27 & 0.22 & 0.38 & 0.50 & 0.32 & 0.26 & 0.37 & 0.35 & 0.31 & 0.32 & 0.28 & 0.36 & 0.81 \\
\hline P2O5 & 39.44 & 39.89 & 39.39 & 39.33 & 39.54 & 39.92 & 39.34 & 39.53 & 39.26 & 39.35 & 39.77 & 39.58 & 40.28 \\
\hline $\mathrm{Cl}$ & 0.04 & 0.03 & 0.03 & 0.03 & 0.03 & 0.03 & 0.04 & 0.04 & 0.03 & 0.03 & 0.03 & 0.04 & 0.05 \\
\hline $\mathrm{CaO}$ & 55.26 & 55.26 & 55.26 & 55.26 & 55.26 & 55.26 & 55.26 & 55.26 & 55.26 & 55.26 & 55.26 & 55.26 & 55.26 \\
\hline$M n$ & 345.30 & 238.20 & 335.06 & 346.31 & 360.46 & 343.31 & 322.18 & 349.50 & 359.69 & 356.17 & 326.12 & 391.90 & 281.34 \\
\hline Sr & 576.64 & 619.75 & 568.55 & 564.11 & 574.81 & 583.11 & 564.15 & 561.64 & 558.99 & 558.86 & 578.54 & 578.26 & 587.13 \\
\hline $\mathbf{Y}$ & 114.27 & 113.00 & 202.22 & 189.45 & 171.24 & 95.53 & 196.76 & 186.11 & 158.12 & 154.03 & 48.47 & 243.08 & 140.02 \\
\hline La & 1368.36 & 1215.09 & 1950.08 & 1930.29 & 1941.57 & 1112.48 & 2124.32 & 2119.28 & 1733.97 & 1753.17 & 548.27 & 2298.77 & 1267.63 \\
\hline $\mathrm{Ce}$ & 1676.69 & 1507.46 & 2442.23 & 2601.00 & 2407.10 & 1373.17 & 2598.03 & 2584.49 & 2099.73 & 2145.62 & 642.11 & 2896.37 & 1553.63 \\
\hline $\mathrm{Pr}$ & 125.42 & 118.31 & 195.74 & 222.15 & 189.21 & 108.14 & 211.70 & 213.70 & 171.60 & 177.44 & 52.27 & 241.10 & 126.61 \\
\hline Nd & 413.22 & 392.26 & 671.81 & 760.24 & 644.49 & 367.68 & 717.16 & 702.43 & 591.84 & 561.99 & 181.26 & 817.23 & 453.91 \\
\hline Sm & 46.56 & 52.59 & 85.34 & 90.52 & 75.15 & 43.60 & 81.60 & 80.76 & 77.18 & 74.96 & 18.87 & 110.43 & 56.11 \\
\hline $\mathrm{Eu}$ & 13.06 & 11.57 & 19.93 & 18.81 & 20.80 & 11.99 & 24.10 & 22.94 & 18.30 & 17.94 & 6.39 & 20.16 & 12.50 \\
\hline Gd & 39.11 & 44.31 & 69.69 & 56.93 & 62.40 & 37.46 & 64.29 & 57.46 & 53.21 & 55.98 & 13.92 & 73.57 & 46.62 \\
\hline $\mathrm{Tb}$ & 4.30 & 3.77 & 5.97 & 6.44 & 6.75 & 3.76 & 7.72 & 7.68 & 5.89 & 5.46 & 1.60 & 7.69 & 4.39 \\
\hline Dy & 20.63 & 27.74 & 38.24 & 35.23 & 42.08 & 19.31 & 39.31 & 38.45 & 25.65 & 27.13 & 9.93 & 47.92 & 23.38 \\
\hline Ho & 3.45 & 3.74 & 6.51 & 6.20 & 5.32 & 3.32 & 6.92 & 5.60 & 5.36 & 6.31 & 2.43 & 6.69 & 5.10 \\
\hline $\mathrm{Er}$ & 11.97 & 9.66 & 17.30 & 18.07 & 12.29 & 10.65 & 12.97 & 13.85 & 12.65 & 14.74 & 6.48 & 27.48 & 16.95 \\
\hline $\mathrm{Tm}$ & 1.32 & 1.45 & 2.29 & 2.58 & 1.42 & 0.91 & 2.45 & 1.96 & 2.45 & 1.63 & 0.64 & 2.68 & 2.34 \\
\hline $\mathbf{Y b}$ & 8.72 & 7.46 & 14.97 & 16.07 & 14.03 & 8.63 & 12.42 & 14.25 & 8.64 & 14.30 & 4.40 & 19.09 & 15.93 \\
\hline Lu & 1.29 & 1.50 & 2.74 & 2.73 & 1.50 & 1.55 & 2.23 & 1.88 & 1.61 & 1.43 & 0.30 & 2.63 & 3.89 \\
\hline Th & 74.74 & 107.19 & 111.38 & 76.55 & 81.89 & 81.65 & 87.98 & 76.16 & 57.38 & 73.28 & 21.89 & 76.01 & 123.87 \\
\hline U & 36.67 & 54.39 & 47.86 & 26.99 & 30.13 & 34.00 & 27.14 & 25.97 & 34.25 & 33.33 & 9.20 & 38.40 & 67.89 \\
\hline $\mathrm{Sr} / \mathrm{Sm}$ & 12.39 & 11.78 & 6.66 & 6.23 & 7.65 & 13.38 & 6.91 & 6.95 & 7.24 & 7.46 & 30.66 & 5.24 & 10.46 \\
\hline $\mathrm{Ce} / \mathrm{Y}$ & 14.67 & 13.34 & 12.08 & 13.73 & 14.06 & 14.37 & 13.20 & 13.89 & 13.28 & 13.93 & 13.25 & 11.92 & 11.10 \\
\hline
\end{tabular}

576.6

2 o $\quad 32.7$

$\mathrm{Ce} / \mathrm{Y}$ avge $\quad 14.7$

2 o 2.1 


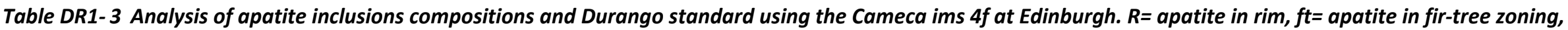
cr= inclusions close by crack. F, SiO2, P2O5, $\mathrm{Cl}$ and $\mathrm{CaO}$ are in wt\%. Other elements are in ppm.s

\begin{tabular}{|c|c|c|c|c|c|c|c|c|c|c|c|c|c|}
\hline & \multicolumn{4}{|c|}{ RGH-1 } & \multicolumn{6}{|c|}{ RT1 } & \multicolumn{3}{|c|}{$\mathbf{R 2}$} \\
\hline & \multicolumn{3}{|c|}{ In titanite } & \multirow{2}{*}{$\begin{array}{c}\text { In zircon } \\
146-78\end{array}$} & \multicolumn{5}{|c|}{ In titanite } & \multirow{2}{*}{$\begin{array}{c}\text { In zircon } \\
163-81\end{array}$} & \multicolumn{2}{|c|}{ In titanite } & \multirow{2}{*}{$\begin{array}{c}\text { In zircon } \\
93-83\end{array}$} \\
\hline & $120-27$ & $r-120-29$ & $r-125-30$ & & $r-7-24$ & $r-7-25$ & ft-cr76-37 & ft-85-38 & ft-85-39 & & $132-40$ & $5-26$ & \\
\hline $\mathbf{F}$ & 2.63 & 2.91 & 3.25 & 2.80 & 2.67 & 2.81 & 2.93 & 2.36 & 2.39 & 2.59 & 2.54 & 2.32 & 2.73 \\
\hline SiO2 & 0.40 & 0.29 & 0.44 & 0.35 & 0.84 & 0.30 & 0.27 & 0.34 & 0.35 & 4.03 & 0.25 & 0.28 & 4.99 \\
\hline P2O5 & 39.57 & 39.97 & 40.47 & 36.38 & 38.92 & 39.64 & 39.93 & 38.65 & 38.96 & 38.65 & 39.01 & 39.29 & 38.27 \\
\hline $\mathrm{Cl}$ & 0.12 & 0.02 & 0.04 & 0.25 & 0.05 & 0.04 & 0.04 & 0.14 & 0.14 & 0.11 & 0.04 & 0.16 & 0.09 \\
\hline $\mathrm{CaO}$ & 55.12 & 55.12 & 55.12 & 55.12 & 55.21 & 55.21 & 55.21 & 55.21 & 55.21 & 55.21 & 55.13 & 55.13 & 55.13 \\
\hline$M n$ & 409.72 & 348.24 & 390.23 & 381.29 & 307.24 & 295.40 & 318.44 & 383.50 & 387.22 & 367.05 & 393.79 & 732.27 & 376.96 \\
\hline $\mathrm{Sr}$ & 819.82 & 719.59 & 710.04 & 904.33 & 924.35 & 927.48 & 945.13 & 1044.89 & 1041.78 & 1036.16 & 679.91 & 763.18 & 723.18 \\
\hline $\mathbf{Y}$ & 171.27 & 250.53 & 363.77 & 281.44 & 456.70 & 248.64 & 314.11 & 398.64 & 400.15 & 492.50 & 230.22 & 540.71 & 508.90 \\
\hline La & 585.23 & 815.60 & 1198.58 & 1319.59 & 939.52 & 544.82 & 658.58 & 1125.90 & 1105.54 & 868.26 & 312.21 & 597.13 & 1078.08 \\
\hline $\mathrm{Ce}$ & 1241.19 & 1719.46 & 2664.88 & 2583.02 & 2373.95 & 1367.13 & 1671.73 & 2663.58 & 2633.08 & 2257.13 & 949.73 & 1740.76 & 2512.07 \\
\hline Pr & 140.72 & 185.54 & 304.44 & 278.52 & 292.19 & 169.06 & 211.57 & 319.02 & 315.59 & 302.57 & 127.47 & 239.34 & 297.87 \\
\hline Nd & 540.74 & 692.98 & 1166.25 & 1059.24 & 1206.60 & 720.11 & 876.93 & 1260.15 & 1231.96 & 1333.38 & 538.69 & 1056.52 & 1274.05 \\
\hline Sm & 76.66 & 97.27 & 155.29 & 132.31 & 193.76 & 102.36 & 123.41 & 180.08 & 173.62 & 223.67 & 99.90 & 197.15 & 181.31 \\
\hline $\mathrm{Eu}$ & 16.75 & 26.76 & 35.28 & 23.61 & 46.86 & 26.68 & 39.30 & 43.17 & 43.80 & 45.85 & 17.29 & 29.30 & 35.45 \\
\hline Gd & 53.07 & 77.92 & 119.37 & 88.80 & 136.22 & 74.43 & 99.17 & 128.72 & 127.35 & 161.37 & 69.64 & 156.20 & 142.16 \\
\hline $\mathrm{Tb}$ & 4.22 & 6.89 & 10.52 & 8.75 & 11.34 & 6.24 & 7.94 & 10.48 & 10.19 & 11.54 & 5.76 & 12.08 & 10.12 \\
\hline Dy & 25.01 & 42.13 & 63.97 & 53.45 & 75.11 & 46.09 & 57.57 & 67.49 & 71.51 & 90.16 & 42.47 & 88.05 & 64.93 \\
\hline Ho & 6.22 & 8.39 & 12.05 & 10.29 & 14.64 & 8.87 & 11.32 & 13.28 & 13.13 & 16.09 & 7.93 & 17.90 & 14.42 \\
\hline $\mathrm{Er}$ & 14.62 & 21.53 & 29.43 & 21.72 & 37.03 & 18.00 & 24.50 & 32.57 & 38.91 & 43.69 & 18.20 & 46.65 & 49.07 \\
\hline $\mathrm{Tm}$ & 1.96 & 3.00 & 3.91 & 4.01 & 5.67 & 3.16 & 3.61 & 4.94 & 5.10 & 6.34 & 2.85 & 4.64 & 6.97 \\
\hline Yb & 9.10 & 18.47 & 26.08 & 18.41 & 30.29 & 18.03 & 22.78 & 28.16 & 33.35 & 50.43 & 14.12 & 37.42 & 52.51 \\
\hline Lu & 1.41 & 3.06 & 3.10 & 1.82 & 4.95 & 2.56 & 4.47 & 5.05 & 5.23 & 11.72 & 2.87 & 4.59 & 11.56 \\
\hline Th & 20.77 & 44.79 & 87.90 & 61.66 & 76.68 & 64.98 & 85.92 & 61.06 & 64.79 & 65.14 & 22.07 & 51.53 & 79.84 \\
\hline $\mathbf{U}$ & 13.03 & 42.37 & 42.56 & 42.57 & 24.82 & 61.93 & 85.79 & 46.07 & 40.86 & 100.20 & 30.92 & 17.68 & 71.77 \\
\hline $\mathrm{Sr} / \mathrm{Sm}$ & 10.69 & 7.40 & 4.57 & 6.83 & 4.77 & 9.06 & 7.66 & 5.80 & 6.00 & 4.63 & 6.81 & 3.87 & 3.99 \\
\hline $\mathrm{Ce} / \mathrm{Y}$ & 7.25 & 6.86 & 7.33 & 9.18 & 5.20 & 5.50 & 5.32 & 6.68 & 6.58 & 4.58 & 4.13 & 3.22 & 4.94 \\
\hline Sr avge & 819.8 & & & & 924.3 & & & & & & 679.9 & & \\
\hline $2 \sigma$ & 183.7 & & & & 120.0 & & & & & & 83.3 & & \\
\hline Ce/Y avge & 7.2 & & & & 5.2 & & & & & & 4.1 & & \\
\hline $2 \sigma$ & 2.1 & & & & 1.6 & & & & & & 1.7 & & \\
\hline
\end{tabular}


Table DR1- 4 Analysis of Durango standard using the Cameca ims $4 f$ at Edinburgh*. F, SiO2, P2O5, Cl and CaO are in wt\%. Other elements are in ppm.

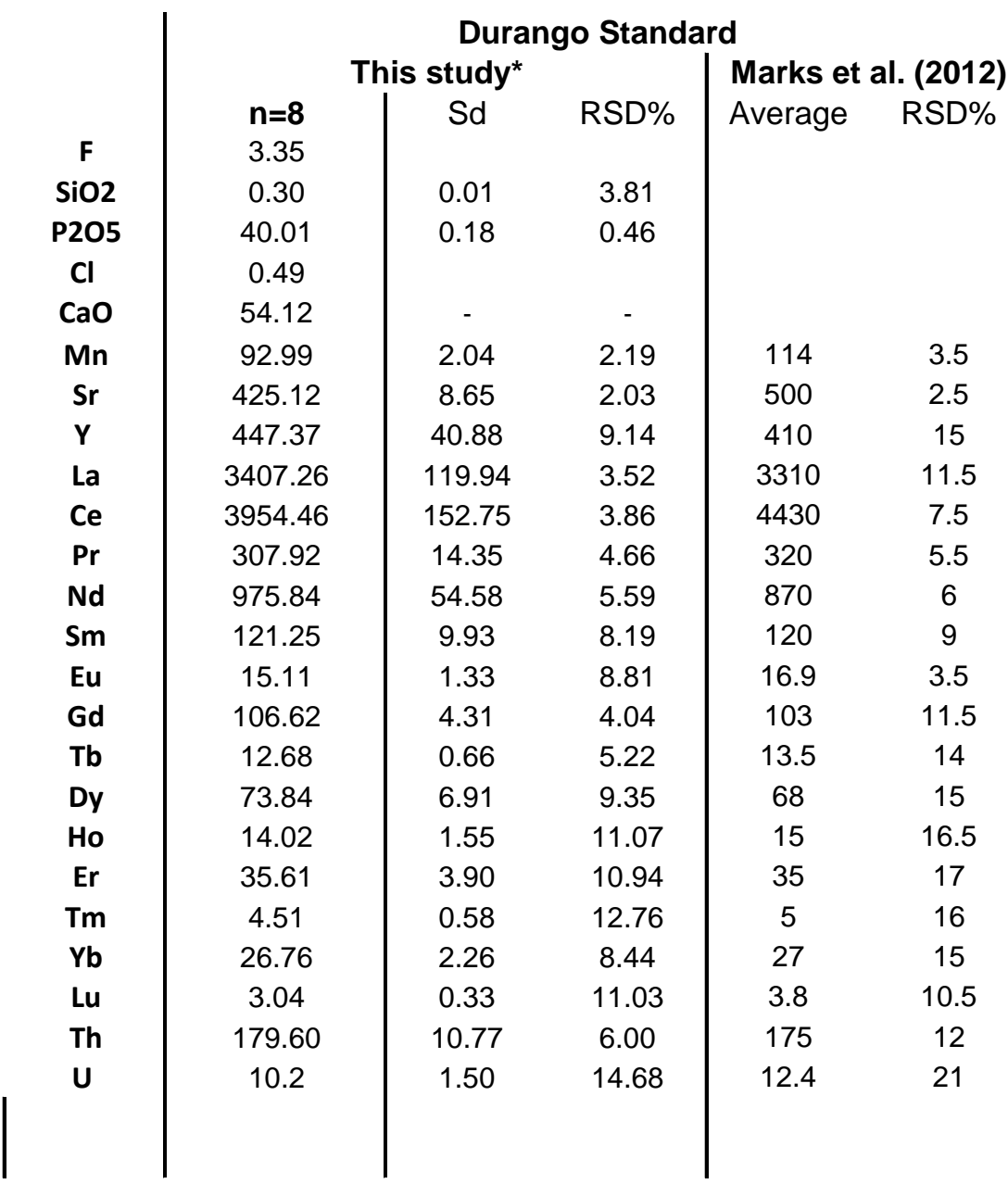


Table DR1- 5 Analysis of apatite inclusions from two sanukitoids (Karelian Province, Finland) using the Cameca SX-100 microprobe at Bristol ${ }^{\#}$. All elemens are in wt\% except when stated(ppm). $Y$ was remove from analysis as Durango standard values for $Y$ (Table DR1-6) were found far off the published value.

\begin{tabular}{|c|c|c|c|c|c|c|c|c|c|c|c|}
\hline & & Sanu & 316 & & & & & A572 & & & \\
\hline & 112 & 123 & 127 & 123 & 52 & $\mathrm{~T} 2$ & T2-2 & T8 & T8-2 & T8-3 & $\mathrm{T} 14$ \\
\hline $\mathbf{F}$ & 3.16 & 2.87 & 2.59 & 2.55 & 3.29 & 2.70 & 2.70 & 2.73 & 2.67 & 2.76 & 2.55 \\
\hline $\mathrm{Na2O}$ & - & 0.05 & 0.04 & 0.03 & 0.04 & 0.16 & 0.13 & 0.08 & 0.12 & 0.28 & 0.18 \\
\hline $\mathrm{SiO} 2$ & 0.16 & 0.24 & 0.31 & 0.34 & 0.28 & 0.23 & 0.20 & 0.21 & 0.24 & 0.38 & 0.21 \\
\hline P2O5 & 41.62 & 41.97 & 40.95 & 41.75 & 40.59 & 40.76 & 41.78 & 42.58 & 41.61 & 39.98 & 41.51 \\
\hline SO2 & 0.10 & 0.17 & - & 0.02 & 0.05 & 0.51 & 0.46 & 0.31 & 0.48 & 0.83 & 0.53 \\
\hline $\mathrm{CaO}$ & 55.40 & 55.79 & 54.46 & 56.02 & 54.24 & 54.63 & 55.82 & 56.05 & 55.70 & 54.41 & 55.46 \\
\hline Cl & 0.04 & 0.03 & 0.01 & 0.00 & 0.04 & 0.02 & 0.02 & 0.03 & 0.02 & 0.04 & 0.02 \\
\hline $\mathrm{TiO} 2$ & - & - & - & - & - & 0.17 & 0.18 & 0.09 & 0.09 & 0.15 & 0.25 \\
\hline MnO & 0.02 & 0.03 & 0.07 & 0.06 & 0.03 & 0.03 & 0.04 & 0.04 & 0.04 & 0.03 & 0.04 \\
\hline $\mathrm{FeO}$ & 0.01 & - & 0.01 & 0.00 & 0.08 & 0.02 & 0.04 & 0.04 & 0.04 & 0.07 & 0.03 \\
\hline Y2O3 & 0.00 & 0.05 & 0.13 & 0.16 & 0.08 & 0.03 & 0.01 & 0.03 & 0.02 & 0.03 & 0.05 \\
\hline SrO & 0.11 & 0.11 & 0.08 & 0.08 & 0.09 & 0.06 & 0.09 & 0.08 & 0.06 & 0.07 & 0.12 \\
\hline Sr (ppm) & 921.69 & 952.13 & 716.21 & 717.91 & 755.96 & 519.19 & 738.20 & 647.72 & 546.25 & 585.15 & 1004.56 \\
\hline $\mathrm{Nd} 2 \mathrm{O} 3$ & 0.11 & 0.16 & 0.18 & 0.17 & 0.10 & 0.04 & 0.02 & 0.01 & 0.03 & 0.05 & 0.09 \\
\hline $\mathrm{Ce} 2 \mathrm{O} 3$ & 0.04 & 0.15 & 0.23 & 0.16 & 0.11 & 0.07 & 0.01 & 0.03 & 0.08 & 0.05 & 0.10 \\
\hline La2O3 & 0.03 & 0.00 & - & 0.06 & 0.04 & 0.02 & 0.05 & 0.04 & 0.05 & - & 0.02 \\
\hline Total & 100.81 & 101.62 & 99.06 & 101.42 & 99.05 & 99.46 & 101.53 & 102.33 & 101.24 & 99.13 & 101.17 \\
\hline SiO2 WR & 64.69 & 64.69 & 64.69 & 64.69 & 67.36 & 67.36 & 67.36 & 67.36 & 67.36 & 67.36 & 67.36 \\
\hline
\end{tabular}

Table DR1- 1 Durango stc $\mathrm{Na2O}$ $\mathrm{SiO} 2$ P2O5

SO2

$\mathrm{CaO}$

CI

TiO2

$\mathrm{MnO}$

$\mathrm{FeO}$

Y2O3

Y(ppm)

$\mathrm{SrO}$

Sr(ppm)

$\mathrm{Nd} 2 \mathrm{O} 3$

$\mathrm{Nd}$ (ppm)

Ce2O3

$\mathrm{Ce}(\mathrm{ppm})$

La2O3

La(ppm)

Total 
5 Analysis of Durango standard using the Cameca SX-100 microprobe at Bristol ${ }^{\#}$. All elemens are in wt\% except when stated(ppm). Y was remove as Indard values for $Y$ are much higher than published values (Table DR1-4, Marks et al., 2012)

\begin{tabular}{|c|c|c|}
\hline \multicolumn{2}{|c|}{$\begin{array}{l}\text { Durango Standard } \\
\text { This study }\end{array}$} & \multirow[b]{2}{*}{ RSD } \\
\hline$n=8$ & Sd & \\
\hline 3.308 & 0.14 & 4.24 \\
\hline 0.178 & 0.04 & 20.07 \\
\hline 0.325 & 0.04 & 12.41 \\
\hline 41.174 & 0.56 & 1.36 \\
\hline 0.236 & 0.02 & 10.60 \\
\hline 54.435 & 0.52 & 0.96 \\
\hline 0.459 & 0.03 & 6.99 \\
\hline 0.003 & 0.00 & 95.24 \\
\hline 0.008 & 0.00 & 49.15 \\
\hline 0.022 & 0.02 & 84.06 \\
\hline 0.078 & 0.00 & 3.03 \\
\hline 613.32 & 18.57 & \\
\hline 0.056 & 0.01 & 12.04 \\
\hline 476.13 & 57.48 & \\
\hline 0.108 & 0.02 & 15.98 \\
\hline 927.97 & 148.28 & \\
\hline 0.500 & 0.05 & 9.66 \\
\hline 4266.61 & 412.20 & \\
\hline 0.384 & 0.06 & 15.5 \\
\hline 3278.45 & 509.34 & \\
\hline 101.27 & & \\
\hline
\end{tabular}


Table DR2- LA-ICPMS analysis for host titanites from Strontian and Rogart samples. Analyses spots are reported in Figure DR1.Numbers in brackets associated with crystal numbers correspond to $4 f$ data (Table DR1). SiO2, P2O5, CaO, TiO2 are in Wt\%. Others are in ppm.

\begin{tabular}{|c|c|c|c|c|c|c|c|c|c|c|c|c|c|c|c|c|}
\hline & Crystal Nb & $\mathrm{SiO}_{2}$ & $\mathrm{P}_{2} \mathrm{O}_{5}$ & $\mathrm{CaO}$ & $\mathrm{TiO}_{2}$ & V & $\mathrm{Cr}$ & $\mathrm{Sr}$ & $\mathbf{Y}$ & $\mathrm{Zr}$ & $\mathrm{Nb}$ & La & $\mathrm{Ce}$ & $\mathrm{Pr}$ & Nd & Sm \\
\hline \multicolumn{17}{|c|}{ Host titanite analysis } \\
\hline \multicolumn{17}{|l|}{ SR3 } \\
\hline AP3E03 & $22(16)$ & 32.4 & & 27.9 & 33.9 & 716.0 & 119.0 & 62.8 & 630.0 & 470.0 & 869.0 & 2920.0 & 5560.0 & 530.0 & 1880.0 & 282.0 \\
\hline NO25E04 & 24 (19) & 35.8 & - & 27.9 & 34.3 & 680.0 & 95.8 & 63.9 & 706.8 & 449.3 & 780.4 & 2623.1 & 5114.4 & 498.1 & 1789.9 & 290.0 \\
\hline NO25E05 & 24 & 36.3 & - & 27.4 & 33.9 & 797.1 & 149.3 & 65.5 & 1874.8 & 568.1 & 1399.7 & 3382.7 & 11014.0 & 1491.5 & 6209.5 & 1058.2 \\
\hline NV26B07 & $4(45,48)$ & 33.6 & - & 27.6 & 34.3 & 677.2 & 92.2 & 76.3 & 786.4 & 686.9 & 2379.7 & 5280.3 & 11996.9 & 1108.0 & 3517.3 & 415.1 \\
\hline NV26B08 & $10(43)$ & 34.6 & - & 27.7 & 33.2 & 748.1 & 153.5 & 69.5 & 990.0 & 577.2 & 1608.2 & 4100.0 & 10910.6 & 1163.7 & 4041.7 & 536.8 \\
\hline \multicolumn{17}{|l|}{ SR4 } \\
\hline NO25E06 & $15(21)$ & 34.6 & - & 27.3 & 33.7 & 744.4 & 132.5 & 65.0 & 3041.1 & 615.3 & 1384.8 & 3696.4 & 12456.4 & 1726.1 & 7300.0 & 1327.0 \\
\hline NO25E08 & $17(22)$ & 35.1 & - & 28.2 & 34.4 & 665.9 & 111.2 & 64.9 & 910.4 & 504.3 & 1158.2 & 2760.7 & 6411.2 & 640.1 & 2187.2 & 308.3 \\
\hline NV26D05 & $50(57)$ & 34.6 & - & 27.3 & 33.4 & 728.7 & 120.3 & 67.0 & 1860.7 & 503.4 & 1542.8 & 3517.5 & 11220.6 & 1325.5 & 5109.3 & 827.3 \\
\hline \multicolumn{17}{|l|}{ SR1 } \\
\hline NO25F04 & $38(2-3)$ & 33.9 & - & 27.0 & 33.1 & 742.0 & 134.4 & 65.2 & 1194.6 & 561.3 & 1293.4 & 3773.5 & 10952.0 & 1271.7 & 4562.4 & 638.1 \\
\hline NO25F07 & $40(13)$ & 33.8 & - & 27.2 & 33.0 & 729.2 & 147.3 & 64.7 & 1317.1 & 628.0 & 1360.7 & 3694.5 & 11280.0 & 1329.3 & 4933.8 & 706.6 \\
\hline NO25F08 & 40 (14) & 34.5 & - & 28.0 & 34.2 & 731.2 & 130.9 & 67.4 & 594.1 & 516.9 & 789.9 & 3169.6 & 7324.2 & 691.2 & 2242.0 & 284.6 \\
\hline NO25F12 & 43 (11) & 32.7 & - & 27.6 & 32.6 & 758.1 & 154.7 & 64.4 & 1298.7 & 537.8 & 985.3 & 3507.3 & 10421.4 & 1236.0 & 4614.0 & 666.0 \\
\hline NO25F14 & 44 (7) & 34.4 & - & 27.6 & 32.9 & 789.1 & 161.0 & 66.2 & 1778.1 & 555.3 & 1184.5 & 3505.9 & 11290.2 & 1465.9 & 6003.3 & 976.9 \\
\hline NV26D09 & $36(51,56)$ & 32.6 & - & 27.3 & 33.7 & 713.3 & 136.8 & 65.4 & 825.7 & 534.1 & 1171.6 & 3754.2 & 9143.1 & 956.0 & 3254.7 & 399.9 \\
\hline \multicolumn{17}{|l|}{ R2 } \\
\hline NO25F15 & $5(26)$ & 34.61 & - & 32.92 & 27.61 & 643.4 & 125.5 & 49.9 & 1553.0 & 293.4 & 1360.3 & 1314.4 & 5372.5 & 777.1 & 3387.1 & 630.1 \\
\hline \multicolumn{17}{|l|}{ RHG1 } \\
\hline MR8A05 & $120(27)$ & 27.7 & - & 27.3 & 30.6 & 751.0 & 159.0 & 88.5 & 2300.0 & 511.0 & 1080.0 & 3440.0 & 11500.0 & 1680.0 & 7380.0 & 1330.0 \\
\hline MR8A07 & 120 (29) & 27.9 & & 27.3 & 30.0 & 783.0 & 186.0 & 82.2 & 2080.0 & 491.0 & 1160.0 & 3160.0 & 10400.0 & 1480.0 & 6320.0 & 1100.0 \\
\hline MR8B14 & $125(30)$ & 27.6 & - & 27.1 & 30.1 & 858.0 & 250.0 & 79.9 & 2480.0 & 489.0 & 1320.0 & 2590.0 & 9370.0 & 1470.0 & 6610.0 & 1250.0 \\
\hline \multicolumn{17}{|l|}{ RT1 } \\
\hline AP3A03 & $7(24)$ & 32.3 & - & 27.3 & 34.2 & 845.0 & 143.0 & 85.9 & 2360.0 & 519.0 & 1310.0 & 2930.0 & 10200.0 & 1520.0 & 6720.0 & 1230.0 \\
\hline AP3A04 & $7(25)$ & 32.9 & - & 28.0 & 34.4 & 843.0 & 227.0 & 83.6 & 1990.0 & 495.0 & 1130.0 & 2550.0 & 8430.0 & 1200.0 & 5100.0 & 885.0 \\
\hline NV26C09 & $85(38-39)$ & 32.4 & - & 27.0 & 32.9 & 648.7 & 143.4 & 116.3 & 1315.6 & 305.4 & 1701.4 & 1698.3 & 6352.9 & 911.9 & 3923.8 & 711.6 \\
\hline NV26C10 & 76 (37) & 33.6 & - & 27.4 & 33.6 & 769.8 & 163.7 & 95.3 & 1654.6 & 420.7 & 1535.3 & 2836.2 & 9968.9 & 1331.7 & 5395.2 & 930.5 \\
\hline
\end{tabular}


Table DR2- LA-ICPMS analysis for host titanites from Strontian and Rogart samples. Analyses spots are reported in Figure DR1. Numbers in brackets associated with crystal numbers correspond to $4 \mathrm{f}$ data (Table DR1). SiO2, P2O5, CaO, TiO2 are in Wt\%. Others are in ppm.

\begin{tabular}{|c|c|c|c|c|c|c|c|c|c|c|c|c|c|c|c|}
\hline & Eu & Gd & Tb & Dy & Ho & $\mathrm{Er}$ & $\mathrm{Tm}$ & $\mathrm{Yb}$ & Lu & $\mathrm{Hf}$ & $\mathrm{Ta}$ & $\mathbf{P b}$ & Th & U & $\mathrm{Nb} / \mathrm{Ta}$ \\
\hline \multicolumn{16}{|c|}{ Host titanite analysis } \\
\hline \multicolumn{16}{|c|}{ SR3 } \\
\hline AP3E03 & 66.1 & 199.0 & 24.9 & 129.0 & 23.5 & 61.4 & 8.4 & 60.8 & 9.0 & 30.9 & 33.5 & - & - & - & - \\
\hline NO25E04 & 72.6 & 212.0 & 27.4 & 142.8 & 25.7 & 66.4 & 9.5 & 70.8 & 10.2 & - & 27.3 & - & - & - & - \\
\hline NO25E05 & 177.4 & 672.4 & 83.0 & 407.7 & 71.6 & 177.6 & 24.6 & 169.4 & 19.5 & - & 174.5 & - & - & - & - \\
\hline NV26B07 & 87.9 & 291.3 & 28.8 & 142.3 & 27.2 & 75.5 & 10.9 & 86.5 & 12.1 & - & 276.0 & - & - & - & - \\
\hline NV26B08 & 103.2 & 361.2 & 37.4 & 185.6 & 34.5 & 91.9 & 13.5 & 102.1 & 13.4 & - & 192.1 & - & - & - & - \\
\hline \multicolumn{16}{|l|}{ SR4 } \\
\hline NO25E06 & 204.0 & 928.8 & 127.0 & 667.4 & 118.0 & 296.9 & 39.9 & 259.5 & 29.2 & - & 178.7 & - & - & - & - \\
\hline NO25E08 & 80.6 & 238.5 & 29.9 & 162.0 & 32.0 & 87.8 & 13.9 & 104.9 & 15.0 & - & 84.7 & - & - & - & - \\
\hline NV26D05 & 155.3 & 573.9 & 74.8 & 393.5 & 72.1 & 179.6 & 25.5 & 177.0 & 20.3 & - & 171.8 & - & - & - & - \\
\hline \multicolumn{16}{|l|}{ SR1 } \\
\hline NO25F04 & 120.7 & 413.3 & 46.6 & 232.6 & 43.3 & 114.1 & 16.6 & 120.4 & 14.8 & - & 148.0 & - & - & - & - \\
\hline NO25F07 & 133.6 & 463.0 & 53.7 & 269.6 & 48.3 & 126.4 & 18.5 & 132.9 & 15.7 & - & 192.5 & - & - & - & - \\
\hline NO25F08 & 66.3 & 200.8 & 21.5 & 107.8 & 20.3 & 55.8 & 8.4 & 66.9 & 9.8 & - & 51.9 & - & - & - & - \\
\hline NO25F12 & 125.7 & 432.3 & 50.3 & 245.7 & 46.7 & 120.7 & 17.9 & 128.8 & 15.8 & - & 112.3 & - & - & - & - \\
\hline NO25F14 & 172.9 & 636.0 & 75.2 & 383.4 & 68.5 & 169.0 & 23.7 & 167.7 & 19.3 & - & 153.1 & - & - & - & - \\
\hline NV26D09 & 79.6 & 268.0 & 29.0 & 146.1 & 28.1 & 76.4 & 11.7 & 91.7 & 12.8 & - & 106.1 & - & - & - & - \\
\hline \multicolumn{16}{|l|}{ R2 } \\
\hline NO25F15 & 135.0 & 436.8 & 56.1 & 297.5 & 56.8 & 146.8 & 21.6 & 149.7 & 18.1 & - & 91.5 & - & - & - & - \\
\hline \multicolumn{16}{|l|}{ RHG1 } \\
\hline MR8A05 & 222.0 & 835.0 & 108.0 & 546.0 & 92.5 & 222.0 & 30.0 & 174.0 & 19.8 & 33.1 & 86.3 & - & - & - & - \\
\hline MR8A07 & 190.0 & 675.0 & 89.7 & 465.0 & 80.8 & 197.0 & 26.6 & 174.0 & 19.8 & 33.0 & 87.3 & - & - & - & - \\
\hline MR8B14 & 208.0 & 785.0 & 107.0 & 550.0 & 96.2 & 236.0 & 31.9 & 214.0 & 23.4 & 31.6 & 115.0 & - & - & - & - \\
\hline \multicolumn{16}{|l|}{ RT1 } \\
\hline AP3A03 & 207.0 & 777.0 & 102.0 & 520.0 & 93.0 & 229.0 & 31.5 & 194.0 & 22.6 & - & 120.0 & - & - & - & - \\
\hline AP3A04 & 164.0 & 579.0 & 76.8 & 398.0 & 74.2 & 190.0 & 26.9 & 184.0 & 22.4 & - & 93.1 & - & - & - & - \\
\hline NV26C09 & 185.5 & 458.8 & 57.2 & 291.8 & 51.9 & 130.4 & 18.4 & 125.2 & 15.5 & - & 122.8 & - & - & - & - \\
\hline NV26C10 & 165.7 & 573.1 & 70.6 & 358.0 & 63.0 & 164.3 & 22.4 & 150.7 & 17.0 & - & 133.0 & - & - & - & - \\
\hline
\end{tabular}


Representative LA-ICPMS analysis for SR4 apatite and titanite matrix. Core (1) and (2) rim of the crystal.

\begin{tabular}{|c|c|c|c|c|c|c|c|c|c|c|c|c|c|c|c|c|}
\hline & & $\mathrm{SiO}_{2}$ & $\mathbf{P}_{2} \mathbf{O}_{5}$ & $\mathrm{CaO}$ & $\mathrm{TiO}_{2}$ & V & $\mathrm{Cr}$ & $\mathrm{Sr}$ & $\mathbf{Y}$ & $\mathrm{Zr}$ & $\mathrm{Nb}$ & La & $\mathrm{Ce}$ & $\mathrm{Pr}$ & Nd & Sm \\
\hline \multicolumn{17}{|l|}{ Titanite } \\
\hline \multicolumn{17}{|l|}{ SR4 } \\
\hline 1-mr07c06 & & 30.1 & - & 28.1 & 31.3 & 774 & 137 & 63.1 & 1450 & 539 & 1280 & 3340 & 8620 & 996 & 3590 & 532 \\
\hline 1-mr07c11 & & 28.1 & - & 27.0 & 30.9 & 847 & 120 & 55.8 & 2950 & 607 & 1510 & 3590 & 11400 & 1600 & 6730 & 1240 \\
\hline 1-mr07c12 & & 28.3 & - & 27.0 & 30.3 & 833 & 127 & 57.9 & 2910 & 628 & 1290 & 3880 & 12300 & 1730 & 7210 & 1320 \\
\hline 1-mr30c05 & & 33.3 & - & 27.0 & 33.9 & 833 & 149 & 66.6 & 3210 & 640 & 1470 & 3590 & 12100 & 1730 & 7520 & 1420 \\
\hline 1-mr30c09 & & 31.6 & - & 27.2 & 33.9 & 757 & 123 & 63.5 & 1620 & 567 & 1490 & 3570 & 9720 & 1140 & 4140 & 625 \\
\hline 1-mr30d05 & & 32.1 & - & 26.7 & 33.1 & 798 & 127 & 65.7 & 2610 & 593 & 1630 & 3650 & 11300 & 1530 & 6350 & 1110 \\
\hline 2-mr07c03 & & 30.5 & - & 28.2 & 32.5 & 780 & 130 & 72.5 & 1260 & 550 & 1960 & 4100 & 9820 & 1060 & 3600 & 505 \\
\hline 2-mr07c04 & & 29.5 & - & 28.4 & 31.5 & 734 & 141 & 65.0 & 890 & 519 & 1050 & 2420 & 5230 & 537 & 1870 & 281 \\
\hline 2-mr30c06 & & 30.9 & - & 28.0 & 34.2 & 703 & 121 & 61.2 & 795 & 514 & 1040 & 2310 & 4820 & 479 & 1660 & 247 \\
\hline 2-mr30c12 & & 33.6 & - & 28.4 & 35.6 & 754 & 116 & 64.4 & 781 & 546 & 1210 & 2190 & 4340 & 417 & 1430 & 215 \\
\hline 2-mr30c13 & & 33.0 & - & 28.0 & 35.4 & 726 & 114 & 63.6 & 751 & 520 & 1120 & 2190 & 4410 & 440 & 1580 & 255 \\
\hline 2-mr30c14 & & 32.7 & - & 27.6 & 36.0 & 697 & 78 & 70.6 & 995 & 589 & 2290 & 3710 & 8010 & 789 & 2700 & 376 \\
\hline 2-mr30d03 & & 32.5 & - & 27.8 & 34.0 & 706 & 124 & 63.3 & 743 & 520 & 1020 & 2240 & 4510 & 445 & 1550 & 241 \\
\hline & $\mathrm{Eu}$ & Gd & $\mathrm{Tb}$ & Dy & Ho & $\mathrm{Er}$ & $\mathrm{Tm}$ & $\mathrm{Yb}$ & Lu & $\mathrm{Hf}$ & $\mathrm{Ta}$ & $\mathrm{Pb}$ & Th & $\mathbf{U}$ & $\mathrm{Nb} / \mathrm{Ta}$ & \\
\hline \multicolumn{17}{|l|}{ Titanite } \\
\hline \multicolumn{17}{|l|}{ SR4 } \\
\hline 1-mr07c06 & 111.0 & 357.0 & 49.9 & 274.0 & 53.3 & 143.0 & 21.3 & 151.0 & 20.4 & 37.4 & 123.0 & 27.2 & 622.0 & 118.0 & 10.4 & \\
\hline 1-mr07c11 & 176.0 & 826.0 & 121.0 & 646.0 & 117.0 & 294.0 & 39.2 & 260.0 & 30.9 & 40.6 & 186.0 & 21.1 & 514.0 & 99.8 & 8.1 & \\
\hline 1-mr07c12 & 184.0 & 907.0 & 125.0 & 678.0 & 119.0 & 291.0 & 39.9 & 244.0 & 28.0 & 45.0 & 167.0 & 24.5 & 554.0 & 94.2 & 7.7 & \\
\hline 1-mr30c05 & 209.0 & 958.0 & 137.0 & 720.0 & 128.0 & 312.0 & 42.6 & 271.0 & 31.0 & 44.7 & 207.0 & 24.9 & 583.0 & 98.5 & 7.1 & \\
\hline 1-mr30c09 & 121.0 & 432.0 & 58.4 & 315.0 & 59.4 & 159.0 & 22.7 & 155.0 & 19.9 & 36.6 & 168.0 & 23.8 & 567.0 & 110.0 & 8.9 & \\
\hline 1-mr30d05 & 178.0 & 775.0 & 107.0 & 569.0 & 102.0 & 253.0 & 34.1 & 207.0 & 24.7 & 38.1 & 197.0 & 24.7 & 591.0 & 106.0 & 8.3 & \\
\hline 2-mr07c03 & 108.0 & 323.0 & 45.0 & 239.0 & 47.3 & 121.0 & 18.2 & 126.0 & 16.5 & 38.6 & 240.0 & 32.8 & 794.0 & 122.0 & 8.2 & \\
\hline 2-mr07c04 & 70.8 & 200.0 & 28.4 & 155.0 & 31.8 & 89.4 & 13.8 & 104.0 & 14.9 & 36.1 & 69.0 & 25.4 & 637.0 & 134.0 & 15.2 & \\
\hline 2-mr30c06 & 64.2 & 191.0 & 25.0 & 141.0 & 27.6 & 76.6 & 11.8 & 91.2 & 13.7 & 34.8 & 62.3 & 25.5 & 633.0 & 130.0 & 16.7 & \\
\hline 2-mr30c12 & 57.6 & 165.0 & 23.0 & 133.0 & 26.3 & 73.3 & 12.0 & 92.0 & 14.3 & 36.5 & 77.5 & 24.4 & 589.0 & 141.0 & 15.6 & \\
\hline 2-mr30c13 & 62.4 & 195.0 & 27.1 & 151.0 & 28.5 & 73.9 & 10.3 & 71.8 & 10.3 & 33.9 & 61.6 & 26.0 & 640.0 & 136.0 & 18.2 & \\
\hline 2-mr30c14 & 95.0 & 280.0 & 35.2 & 191.0 & 37.3 & 95.7 & 13.5 & 93.2 & 13.9 & 45.5 & 297.0 & 43.7 & 1110.0 & 129.0 & 7.7 & \\
\hline 2-mr30d03 & 62.9 & 183.0 & 24.7 & 137.0 & 26.9 & 70.9 & 10.6 & 81.0 & 12.1 & 34.9 & 57.6 & 27.2 & 648.0 & 126.0 & 17.7 & \\
\hline
\end{tabular}


$\begin{array}{lllllllllllllll}\mathrm{SiO}_{2} & \mathrm{P}_{2} \mathrm{O}_{5} & \mathrm{CaO} & \mathrm{TiO}_{2} & \mathrm{~V} & \mathrm{Cr} & \mathrm{Sr} & \mathrm{Y} & \mathrm{Zr} & \mathrm{Nb} & \mathrm{La} & \mathrm{Ce} & \mathrm{Pr} & \mathrm{Nd} & \mathrm{Sm}\end{array}$

\begin{tabular}{|c|c|c|c|c|c|c|c|c|c|c|c|c|c|c|c|c|}
\hline Apatite & & & & & & & & & & & & & & & & \\
\hline \multicolumn{17}{|l|}{ SR4 } \\
\hline 1-ap04g12 & & - & 46.6 & 55.9 & - & 11.2 & - & 659 & 715 & - & - & 1670 & 3340 & 370 & 1450 & 238 \\
\hline 1-ap04g13 & & - & 42.1 & 54.8 & - & 10.2 & - & 652 & 608 & - & - & 1460 & 2890 & 309 & 1220 & 208 \\
\hline 1-ap04g14 & & - & 43.3 & 54.8 & - & 26.5 & - & 659 & 651 & - & - & 2000 & 3500 & 363 & 1320 & 215 \\
\hline 1-ap04h07 & & - & 45.3 & 54.8 & - & 10.8 & - & 651 & 447 & - & - & 1840 & 3310 & 308 & 1110 & 153 \\
\hline 1-sp03g04 & & - & 43.5 & 55 & - & 25.8 & - & 669 & 489 & - & - & 1870 & 3320 & 334 & 1140 & 158 \\
\hline 1-sp03g07 & & - & 41.5 & 55 & - & 21.6 & - & 660 & 522 & - & - & 1760 & 2870 & 300 & 1100 & 169 \\
\hline 1-sp03g08 & & - & 42.4 & 55 & - & 9.89 & - & 648 & 600 & - & - & 1930 & 3420 & 342 & 1280 & 200 \\
\hline 1-sp03g09 & & - & 44.5 & 55 & - & 20.4 & - & 659 & 532 & - & - & 2280 & 3730 & 357 & 1200 & 176 \\
\hline 1-sp03g14 & & - & 41.4 & 55 & - & 26.3 & - & 662 & 455 & - & - & 2170 & 3260 & 309 & 1050 & 150 \\
\hline 1-sp04a07 & & - & 44.2 & 55 & - & 23.5 & - & 666 & 697 & - & - & 2760 & 4540 & 437 & 1550 & 230 \\
\hline 1-sp04a09 & & - & 43.6 & 55 & - & 31.1 & - & 658 & 475 & - & - & 2120 & 3370 & 319 & 1070 & 146 \\
\hline 1-sp04a14 & & - & 42.8 & 55 & - & 29 & - & 652 & 705 & - & - & 1820 & 3470 & 371 & 1390 & 221 \\
\hline \multirow[t]{2}{*}{ 1-oc01b05 } & & - & 41.6 & 55 & - & 32.9 & - & 680 & 350 & - & - & 2290 & 3120 & 274 & 916 & 118 \\
\hline & $\mathrm{Eu}$ & Gd & Tb & Dy & Ho & $\mathrm{Er}$ & $\mathrm{Tm}$ & $\mathrm{Yb}$ & Lu & $\mathrm{Hf}$ & $\mathrm{Ta}$ & $\mathrm{Pb}$ & Th & $\mathbf{U}$ & $\mathrm{Nb} / \mathrm{Ta}$ & \\
\hline \multicolumn{17}{|l|}{ Apatite } \\
\hline \multicolumn{17}{|l|}{ SR4 } \\
\hline 1-ap04g12 & 25 & 192 & 24.4 & 129 & 25.5 & 64.4 & 8.54 & 53.8 & 7.99 & - & - & 9.7 & 120 & 41.1 & - & \\
\hline 1-ap04g13 & 21.7 & 162 & 21.4 & 110 & 21.1 & 54.1 & 7.28 & 46.7 & 6.8 & - & - & 8.02 & 75.5 & 25.8 & - & \\
\hline 1-ap04g14 & 28.7 & 155 & 21.2 & 111 & 21.5 & 55.2 & 7.21 & 52 & 7.82 & - & - & 13.1 & 108 & 40 & - & \\
\hline 1-ap04h07 & 26.4 & 124 & 15.8 & 79.5 & 15.8 & 41.6 & 5.82 & 34.9 & 5.98 & - & - & 11.4 & 143 & 77.2 & - & \\
\hline 1-sp03g04 & 25 & 113 & 14.7 & 76.6 & 16.3 & 42.5 & 6.17 & 43.7 & 6.77 & - & - & 10.4 & 71.7 & 21.8 & - & \\
\hline 1-sp03g07 & 22.5 & 126 & 16.3 & 89.3 & 16.7 & 44.4 & 6.36 & 41.5 & 6.61 & - & - & 11 & 81.8 & 23.7 & - & \\
\hline 1-sp03g08 & 26.5 & 152 & 19.6 & 106 & 20.9 & 52.1 & 7.35 & 47.7 & 7.34 & - & - & 10.1 & 109 & 55.3 & - & \\
\hline 1-sp03g09 & 28.6 & 130 & 17.2 & 89.3 & 16.9 & 46.9 & 6.14 & 42.3 & 7.01 & - & - & 12.3 & 131 & 50.1 & - & \\
\hline 1-sp03g14 & 24.4 & 106 & 13.6 & 73.5 & 14.2 & 38.6 & 5.49 & 40.7 & 6.21 & - & - & 13.1 & 97.1 & 34.4 & - & \\
\hline 1-sp04a07 & 33.5 & 164 & 21.7 & 118 & 22.6 & 57.6 & 8.68 & 55.6 & 8.74 & - & - & 13.4 & 153 & 50.8 & - & \\
\hline 1-sp04a09 & 26.8 & 101 & 13.3 & 72.4 & 14.4 & 40.1 & 6.32 & 45.5 & 7.24 & - & - & 11.3 & 68.8 & 23.7 & - & \\
\hline 1-sp04a14 & 28.2 & 156 & 21.4 & 116 & 23.2 & 61 & 8.6 & 57.8 & 8.89 & - & - & 11.5 & 85 & 30.3 & - & \\
\hline 1-oc01b05 & 21.3 & 87.7 & 10.4 & 53 & 10.5 & 27.1 & 4.15 & 29.4 & 4.8 & - & - & 10.1 & 61.9 & 19.6 & - & \\
\hline
\end{tabular}




\begin{tabular}{|c|c|c|c|c|c|c|c|c|c|c|c|c|c|c|c|c|}
\hline Apatite & & $\mathrm{SiO}_{2}$ & $\mathrm{P}_{2} \mathrm{O}_{5}$ & $\mathrm{CaO}$ & $\mathrm{TiO}_{2}$ & V & $\mathrm{Cr}$ & $\mathrm{Sr}$ & $\mathbf{Y}$ & $\mathrm{Zr}$ & $\mathrm{Nb}$ & La & $\mathrm{Ce}$ & $\mathrm{Pr}$ & $\mathrm{Nd}$ & Sm \\
\hline 1-oc01b13 & & - & 41.8 & 55 & - & 4.18 & - & 634 & 213 & - & - & 585 & 1190 & 121 & 471 & 72.8 \\
\hline 1-oc01b07 & & - & 43.3 & 55 & - & 28.1 & - & 662 & 576 & - & - & 2100 & 3420 & 343 & 1210 & 179 \\
\hline 1-oc01b08 & & - & 41.9 & 55 & - & 24.6 & - & 637 & 599 & - & - & 1860 & 3450 & 349 & 1230 & 182 \\
\hline 2-ap04h04 & & - & 43.1 & 55.1 & - & 7.94 & - & 663 & 228 & - & - & 981 & 1610 & 149 & 542 & 80.5 \\
\hline 2-ap04h08 & & - & 41.5 & 55.1 & - & 8.43 & - & 651 & 215 & - & - & 888 & 1540 & 146 & 529 & 76.6 \\
\hline 2-sp03g05 & & - & 42.6 & 55 & - & 8.31 & - & 655 & 263 & - & - & 1030 & 1890 & 185 & 645 & 90.5 \\
\hline 2-sp03g06 & & - & 42.5 & 55 & - & 7 & - & 666 & 256 & - & - & 818 & 1520 & 157 & 575 & 91.7 \\
\hline 2-sp03g10 & & - & 42.2 & 55 & - & 8.03 & - & 647 & 222 & - & - & 899 & 1480 & 145 & 521 & 79 \\
\hline 2-sp03g13 & & - & 42.9 & 55 & - & 8.26 & - & 646 & 223 & - & - & 882 & 1510 & 153 & 547 & 78.3 \\
\hline 2-sp04a08 & & - & 44.9 & 55 & - & 7.38 & - & 670 & 281 & - & - & 977 & 1650 & 167 & 632 & 102 \\
\hline 2-sp04a10 & & - & 46.6 & 55 & - & 8.44 & - & 668 & 264 & - & - & 933 & 1730 & 166 & 591 & 87 \\
\hline 2-sp04a11 & & - & 45.9 & 55 & - & 7.66 & - & 646 & 285 & - & - & 991 & 1780 & 176 & 646 & 95.4 \\
\hline 2-sp04a13 & & - & 44.8 & 55 & - & 6.95 & - & 667 & 229 & - & - & 1140 & 1800 & 164 & 576 & 83.7 \\
\hline 2-ap04h05 & & - & 42.7 & 55.2 & - & 6.64 & - & 675 & 250 & - & - & 994 & 1740 & 158 & 599 & 85.2 \\
\hline 2-ap04h06 & & - & 42.1 & 55.4 & - & 1.95 & - & 650 & 332 & - & - & 1320 & 2180 & 209 & 778 & 121 \\
\hline 2-sp04a06 & & - & 43.3 & 55 & - & 4.74 & - & 661 & 225 & - & - & 832 & 1440 & 142 & 511 & 76.4 \\
\hline 2-oc01b04 & & - & 42 & 55 & - & 0.412 & - & 699 & 116 & - & - & 463 & 794 & 78.5 & 291 & 45.5 \\
\hline 2-oc01b09 & & - & 42.7 & 55 & - & 9.26 & - & 650 & 496 & - & - & 1360 & 2700 & 280 & 1070 & 167 \\
\hline 2-oc01b10 & & - & 42.7 & 55 & - & 1.04 & - & 652 & 662 & - & - & 1590 & 2960 & 322 & 1320 & 230 \\
\hline 2-oc01b11 & & - & 43.3 & 55 & - & 4.11 & - & 659 & 274 & - & - & 688 & 1300 & 141 & 566 & 96.2 \\
\hline 2-oc01b12 & & - & 41.3 & 55 & - & 8.4 & - & 616 & 240 & - & - & 693 & 1370 & 140 & 549 & 87.7 \\
\hline 2-oc01b14 & & - & 40.7 & 55 & - & 2.75 & - & 635 & 212 & - & - & 684 & 1320 & 131 & 500 & 75.9 \\
\hline Apatite & $\mathrm{Eu}$ & Gd & $\mathrm{Tb}$ & Dy & Ho & $\mathrm{Er}$ & $\mathrm{Tm}$ & $\mathrm{Yb}$ & Lu & $\mathrm{Hf}$ & $\mathrm{Ta}$ & $\mathrm{Pb}$ & Th & $U$ & $\mathrm{Nb} / \mathrm{Ta}$ & \\
\hline 1-oc01b13 & 10.2 & 58.1 & 7.35 & 37.7 & 7.69 & 19.7 & 2.54 & 14.9 & 2.48 & - & - & 5.4 & 35.5 & 54.4 & - & \\
\hline 1-oc01b07 & 27.4 & 127 & 17.1 & 89 & 17.7 & 48.7 & 7.03 & 47.5 & 7.47 & - & - & 10.6 & 78.3 & 24 & - & \\
\hline 1-oc01b08 & 26.4 & 133 & 16.9 & 92.9 & 17.8 & 49 & 7.07 & 48.3 & 7.77 & - & - & 14.1 & 141 & 72.1 & - & \\
\hline 2-ap04h04 & 12.7 & 60.4 & 7.84 & 40.3 & 7.8 & 19.8 & 2.63 & 17.4 & 2.51 & - & - & 7.99 & 37 & 19.9 & - & \\
\hline 2-ap04h08 & 11.2 & 61.3 & 7.22 & 38.6 & 7.21 & 18.8 & 2.58 & 16.5 & 2.74 & - & - & 7.95 & 40.3 & 21 & - & \\
\hline 2-sp03g05 & 15.1 & 62 & 8.65 & 44.2 & 8.49 & 24.7 & 3.04 & 22.8 & 3.53 & - & - & 5.89 & 53.1 & 52.3 & - & \\
\hline 2-sp03g06 & 13.3 & 70.2 & 8.91 & 47.3 & 9.32 & 23.9 & 3.19 & 19.1 & 3.32 & - & - & 6.62 & 38.8 & 31.7 & - & \\
\hline 2-sp03g10 & 11.5 & 58.3 & 7.18 & 38.1 & 7.52 & 18.8 & 2.5 & 17.4 & 2.62 & - & - & 7.11 & 38.4 & 20.6 & - & \\
\hline 2-sp03g13 & 13.9 & 58.3 & 7.76 & 39.3 & 7.65 & 20.7 & 2.84 & 18 & 3.26 & - & - & 5.95 & 44.8 & 56.1 & - & \\
\hline 2-sp04a08 & 13.3 & 75.7 & 9.88 & 50.1 & 9.95 & 24.9 & 3.35 & 21.1 & 3.37 & - & - & 6.93 & 38.1 & 37.2 & - & \\
\hline
\end{tabular}




\begin{tabular}{|c|c|c|c|c|c|c|c|c|c|c|c|c|c|c|c|}
\hline Apatite & Eu & Gd & Tb & Dy & Ho & $\mathrm{Er}$ & $\mathrm{Tm}$ & $\mathrm{Yb}$ & Lu & $\mathrm{Hf}$ & Ta & $\mathrm{Pb}$ & Th & $\mathbf{U}$ & $\mathrm{Nb} / \mathrm{Ta}$ \\
\hline 2-sp04a10 & 13.1 & 58.5 & 7.95 & 43.9 & 8.46 & 23 & 3.5 & 23.6 & 3.41 & - & - & 6.38 & 37 & 28.8 & - \\
\hline 2-sp04a11 & 14.6 & 70.2 & 9.05 & 48.8 & 9.72 & 24.3 & 3.54 & 24.7 & 3.75 & - & - & 6.14 & 42.7 & 43.4 & - \\
\hline 2-sp04a13 & 13.2 & 57.1 & 7.51 & 39.7 & 7.68 & 19.8 & 2.53 & 18.5 & 2.86 & - & - & 7.49 & 50.2 & 30.6 & - \\
\hline 2-ap04h05 & 12.9 & 69.9 & 8.36 & 45.9 & 8.09 & 22.2 & 3.26 & 17 & 3.05 & - & - & 6.63 & 46.6 & 53 & - \\
\hline 2-ap04h06 & 16.9 & 96.1 & 11.9 & 61.5 & 12 & 29.1 & 3.62 & 26.3 & 4.04 & - & - & 6.98 & 57.4 & 106 & - \\
\hline 2-sp04a06 & 11.7 & 57.9 & 7.56 & 39.8 & 7.83 & 19.7 & 2.57 & 18.4 & 2.85 & - & - & 5.76 & 43.5 & 62.8 & - \\
\hline 2-oc01b04 & 6.46 & 31.9 & 4.07 & 22.2 & 4.18 & 9.79 & 1.37 & 8.28 & 1.18 & - & - & 4.54 & 3.26 & 7.7 & - \\
\hline 2-oc01b09 & 23 & 133 & 17.2 & 88.4 & 17 & 42.9 & 6.08 & 40.7 & 6.41 & - & - & 7.71 & 100 & 80 & - \\
\hline 2-oc01b10 & 26.1 & 191 & 23.7 & 127 & 24.6 & 61.4 & 7.63 & 48.4 & 7.18 & - & - & 8.21 & 83.2 & 190 & - \\
\hline 2-oc01b11 & 11.4 & 81.1 & 10.2 & 50.9 & 9.78 & 25.1 & 3.14 & 18 & 2.72 & - & - & 6.21 & 41.9 & 60.1 & - \\
\hline 2-oc01b12 & 13.3 & 71.5 & 8.85 & 45.7 & 8.57 & 21.6 & 2.57 & 17 & 2.61 & - & - & 5.91 & 44.1 & 74.9 & - \\
\hline 2-oc01b14 & 10.8 & 59.6 & 7.85 & 40.7 & 7.57 & 19.5 & 2.51 & 17.5 & 2.53 & - & - & 5.92 & 42.3 & 65.2 & - \\
\hline
\end{tabular}


Table DR3- Statistic correlations for figure 1 regression lines

Figure 1A

R Square P-value

R Square $\quad 0,8842366641,06852 \mathrm{E}-22$

Figure 1C lower correlation

R square $\quad 0,552177626 \quad 2,21082 \mathrm{E}-09$

Figure 1C upper correlation

R square $\quad 0,534770935 \quad 1,48278 \mathrm{E}-11$

Figure 1D

R Square $\quad 0,620306787 \quad 5,10936 E-11$ 